\title{
Organização da Atenção Primária à Saúde no Paraná no enfrentamento da pandemia Covid-19
}

\author{
Organization of Primary Health Care in Paraná in \\ the pandemic Covid-19
}

\begin{abstract}
Daniel Josivan de Sousa ${ }^{1}$, Carmen Cristina Moura dos Santos², Maria Goretti David Lopes ${ }^{3}$, Sidneya Marques Svierdsovslii ${ }^{4}$
\end{abstract}

\begin{abstract}
1. ORCID: https://orcid.org/0000-0002-0726-4151. Enfermeiro. Divisão Saúde da Família da Secretaria de Estado da Saúde do Paraná, Curitiba, PR, Brasil. Mestre em Saúde e Comunidade - PPGSC/UFPI. E-mail: daniel.josivan@sesa.pr.gov.br.
\end{abstract}

2. ORCID: https://orcid.org/0000-0001-7952-9588. Enfermeira. Coordenadora da Organização de Rede em Cuidados da Saúde da Secretaria de Estado da Saúde do Paraná, Curitiba, PR, Brasil. E-mail: carmen.moura@sesa.pr.gov.br.

3. ORCID: https://orcid.org/0000-0001-7473-6342 Enfermeira. Diretora de Atenção e Vigilância em Saúde da Saúde da Secretaria de Estado da Saúde do Paraná, Curitiba, PR, Brasil. E-mail. maria.lopes@sesa.pr.gov.br.

4. ORCID: https://orcid.org/0000-0002-7102-4865. Enfermeira. Gerente de Atenção Primária à Saúde da Secretaria de Estado da Saúde do Paraná, Curitiba, PR, Brasil. E-mail. sidneyamarques@sesa.pr.gov.br.

CONTATO: Daniel Josivan de Sousa | Endereço: R. Piquiri, 170 - Rebouças, Curitiba - PR, 80230140 - Telefone: (41) 33304278 | E-mail: daniel.josivan@sesa.pr.gov.br

RESUMO A Covid-19 tem representado a maior crise sanitária, econômica e social do novo milênio, caracterizando um desafio global aos sistemas de saúde. O objetivo deste estudo foi descrever as ações e atividades para a organização da atenção primária à saúde no Paraná em resposta à pandemia de Covid-19, segundo o Plano de Contingência Estadual. Trata-se de um estudo descritivo, qualitativo, do tipo relato de experiência. A resposta coordenada do Estado do Paraná frente à Covid-19 partiu do Plano de Resposta a Emergências em Saúde Pública. Fundamentado nesse plano, a Secretaria da Saúde ativou o Comitê de Operações de Emergência em Saúde Pública, publicando posteriormente o Plano de Contingência - Novo Coronavírus, composto por três níveis de resposta. Diante desse risco, configurado na doença Covid-19, a elaboração e atualização do plano de contingência foram fundamentais na organização das ações de intervenção na realidade e geração de respostas 
apropriadas a essa pandemia.

DESCRITORES: Planos de emergência. Infecções por coronavírus. Atenção primária à saúde. Sistema único de saúde.

ABSTRACT Covid-19 has been the biggest health, economic and social crisis in the new millennium, representing a global challenge to health systems. This paper describes actions and activities towards the health primary care in Paraná in response to Covid-19 pandemic, according to the State Contingency Plan. It is a case study, descriptive, qualitative, method. The coordinated answer of Paraná state against Covid-19 started with the Public Health Contingency Plan and, based on it, the Health State Secretariat set in motion a specific Emergency Operations Committee, and afterwards published the Preparedness Plan - New Coronavirus with three levels of response. Given this risk, configured in Covid-19 disease, the elaboration and updating of the contingency plan were fundamental in the organization of intervention actions in reality and generation of appropriate responses to this pandemic.

DESCRIPTORS: Emergency Plans. Coronavirus Infections. Primary Health Care. Unified Health System.

\section{INTRODUÇÃO}

A Covid-19 (Coronavirus Disease 2019), doença emergente infecciosa causada pelo coronavírus, denominado SARS-CoV-2, tem representado a maior crise sanitária, econômica e social do novo milênio, caracterizando um desafio global aos sistemas de saúde. Mundialmente, a pandemia já afetou mais de 216 países e territórios, com registros de 9 milhões de casos confirmados e mais de 469 mil mortes'.

A gravidade e o curso da pandemia fizeram com que muitos governos nacionais adotassem ações estratégicas de redução de riscos, a fim mitigar a propagação do vírus, o acometimento de novos indivíduos e reduzir a sobrecarga social da doença e sua mortalidade ${ }^{2}$. De acordo com estimativas oficiais, $81 \%$ das pessoas acometidas pela Covid-19 podem ser manejadas na Atenção Primária 
à Saúde (APS), por caracterizarem-se como casos leves 3 .

A APS é considerada a principal e mais adequada forma de acesso das pessoas ao sistema de saúde, estando diretamente associada à distribuição mais equitativa da saúde entre populações ${ }^{4-5}$. Dessarte, durante surtos e epidemias e, em especial, em pandemias, a APS tem papel fundamental na resposta global à doença, objetivando atendimento resolutivo, além de manter a longitudinalidade e a coordenação do cuidado em todos os níveis de atenção à saúde, com grande potencial de identificação precoce e encaminhamento de casos graves aos centros de referências ou serviços hospitalares ${ }^{6}$.

Nesse contexto, o presente relato visa a descrever as ações e atividades para a organização da atenção primária à saúde no Paraná em resposta à pandemia Covid-19, segundo o Plano de Contingência do Paraná Covid-19.

\section{MÉTODO}

Trata-se de um estudo descritivo, qualitativo, do tipo relato de experiência.

O Plano de Contingência é um documento que visa a integralidade das ações na prevenção e monitoramento da doença, bem como na assistência à saúde da população. Estas diretrizes têm por objetivo colaborar com os serviços de saúde na mitigação dos processos epidêmicos, comunicação de risco e na redução da morbimortalidade por Covid-19.

Assim, esse documento especifica as medidas a serem adotadas de acordo com a evolução da doença estruturada em três níveis: o nível de resposta 1Atenção, corresponde a uma situação em que o risco de introdução do SARS-COV-2 no Estado do Paraná seja elevado e não apresente casos suspeitos. O nível de resposta 2- Ameaça, já existem casos confirmados laboratorialmente e no nível de resposta 3- Execução, há confirmação de transmissão local no Paraná. 
Direcionado às 22 Regionais de Saúde (RS), que constituem as instâncias administrativas intermediárias da Secretaria de Estado da Saúde (SESA), e as 399 secretarias municipais de saúde, o Plano de Contingência apoia o desenvolvimento de ações de saúde e gestão, fomentando a busca contínua e crescente da eficiência das ações com qualidade.

Por se tratar de um relato de experiência, foi dispensada a apreciação pelo Comitê de Ética em Pesquisa, em conformidade com as diretrizes da Resolução do Conselho Nacional de Saúde (CNS) n 510, de 7 de abril de 2016.

\section{RESULTADOS E DISCUSSÃO}

A pandemia de Covid-19 requer respostas rápidas do Sistema Único de Saúde (SUS) em seu enfrentamento. Nesse período, percorreu-se pelas fases do desconhecimento, formulação de orientações e, atualmente, a fase de implementação de ações, considerando o principal desafio que é garantir o atendimento com qualidade e em tempo oportuno, não apenas relacionadas à Covid-19, como todas as demais necessidades em saúde da população.

Desta forma, a resposta coordenada do Estado do Paraná frente à Covid-19 partiu do Plano de Resposta a Emergências em Saúde Pública7 ${ }^{7}$. Fundamentado nesse plano, a SESA ativou o Comitê de Operações de Emergência em Saúde Pública (COE-Covid-19) ${ }^{8}$, publicando posteriormente o Plano de Contingência - Novo Coronavírus (2019-Ncov) ${ }^{9}$, composto por três níveis de resposta: atenção, ameaça e execução.

O nível de resposta 1- Atenção, corresponde a uma situação em que o risco de introdução do SARS-COV-2 no Estado do Paraná seja elevado e não apresente casos suspeitos.

Nesse documento, foram definidas as responsabilidades do Estado e Municípios, em parceria com o Ministério da Saúde, estabelecendo uma organização necessária, de modo a atender as emergências relacionadas à circulação do vírus Sars-CoV-2. 
A APS organizou-se em oito ações que foram descritas em atividades nos três níveis de resposta, sendo a única diferença entre os níveis a inclusão do monitoramento do caso confirmado a partir do nível 2, conforme descrito abaixo:

1. Padronizar as ações para a detecção precoce de pessoas caracterizadas como casos suspeitos de infecção pelo novo coronavírus (Covid-19);

2. Realizar o manejo clínico dos casos suspeitos de infecção pelo novo coronavírus (Covid-19);

3. Realizar atendimento de forma oportuna e segura, considerando a condição clínica do usuário;

4. Obter informações clínicas, histórico de viagem internacional ou contato com caso suspeito ou confirmado;

5. Apoiar a equipe da vigilância na realização de busca ativa dos contatos;

6. Realizar notificação imediata;

7. Adotar medidas para reduzir casos graves e óbitos;

8. Orientar a população sobre medidas de prevenção e controle.

O nível de resposta 2- Ameaça, corresponde à situação de introdução do Covid-19 no Paraná, ou seja, que os casos suspeitos se tornem confirmados laboratorialmente. Assim, os primeiros casos de Covid-19 no Estado do Paraná foram confirmados em 12 de março, em Curitiba e Cianorte.

A doença se propagou rapidamente. Em menos de quinze dias após a confirmação dos primeiros casos, em 27 de março ocorreu o primeiro óbito por Covid-19 na cidade Maringá - PR.

Diante desse cenário epidemiológico e da declaração da OMS do estado de pandemia, a resposta à Covid-19 passa para o nível de resposta 3- Execução, situação em que há confirmação de transmissão local do primeiro caso do Covid-19, necessitando da mobilização de recursos adicionais e apoio comple- 
mentar, ocorrendo atualização do Plano de Contingência do Paraná Covid-1911.

Assim, não houve alterações nas orientações das ações, contudo, foram incluídas outras atividades como: distanciamento de pelo menos 2 metros, determinado o prazo de monitoramento dos casos por 14 dias, encaminhamento para outros níveis de atenção quando não for possível o isolamento domiciliar devido à condição clínica. Além dessas inclusões, foram realizadas a remoção do histórico de viagem internacional ou contato com caso suspeito ou confirmado das ações de informações clínicas.

Por se tratar de uma doença nova, as lacunas de informação e conhecimento ainda são muito grandes. Diante do desconhecido, surgiu o medo, resultando em ações que acabaram interferindo no atendimento integral e longitudinal dos pacientes, sendo necessário pensar no contexto de garantir o acompanhamento não só dos casos suspeitos e confirmados de Covid-19, mas também de todas as outras condições já existentes antes da pandemia, reorganizando e fortalecendo a APS como porta de entrada na Rede de Atenção à Saúde.

Nesse contexto, o Plano de Contingência do Paraná Covid-1913 passou novamente por atualizações, em 3 junho de 2020, passando de 8 para 10 ações da APS, incluindo assim:

9. Reorganização da Rede de Atenção à Saúde (RAS) nas regiões, realizando o diagnóstico situacional e apoiando as regiões e os municípios no planejamento, organização e acompanhamento das ações da RAS no contexto da pandemia;

10. Apoio aos municípios para a organização da atenção à saúde do idoso.

Essa nova versão elucida a importância da prevenção e controle da Covid-19 às pessoas que são acompanhadas periodicamente pelas equipes da APS. Destacando as atividades:

- Realizar atendimentos na APS observando as medidas de prevenção e as notas orientativas em relação às linhas de atenção: Nota Orientativa 04 
- Atendimento dos idosos frente à pandemia Covid-19; nota Orientativa 09 - Orientações às equipes e profissionais sobre linha de cuidado materno infantil durante a emergência em saúde pública coronavírus Covid-19; nota Orientativa 15 - Saúde Mental; Nota Orientativa 18 - Atendimentos em oncologia frente à pandemia Covid-19; nota Orientativa 29 - Atendimento em puericultura; nota Orientativa 32 - Cuidados às pessoas com Hipertensão Arterial Sistêmica e Diabetes Mellitus frente à Pandemia da Covid-19.

- Acompanhamento longitudinal dos usuários com condições crônicas.

- A implantação de estratégias de acompanhamento e monitoramento do plano de cuidados dos pacientes com condição crônica atendidos pela Atenção Ambulatorial Especializada (AAE).

- A realização esquema vacinal nos grupos vulneráveis a outras doenças respiratórias.

- Acompanhamento dos pacientes confirmados pós alta.

A inclusão das atividades de prevenção e controle da Covid-19 à população acima de 60 anos tem embasamento na alta letalidade nessa faixa etária, visando intensificar as ações de prevenção e controle principalmente nos residentes das Instituições de Longa Permanência de Idosos (ILPI), população esta frágil e com níveis variados de dependência e necessidades complexas.

Assim, o intuito é fomentar a identificação precoce de surtos, para que medidas sejam realizadas em tempo oportuno, prevenindo casos e óbitos. Orientou-se que a partir dessa nova atualização os municípios identifiquem os usuários idosos no território, incluindo os institucionalizados, ILPI, asilos e casas de acolhimento de idosos, estratificando o risco pelo Índice de Vulnerabilidade Social/IVCF-20 e monitorando os idosos frágeis.

Outro ponto importante foi o registro da evolução dos casos no sistema "Notifica Covid-19", assim como o esclarecimento sobre a testagem, princi- 
palmente em relação aos tipos que foram incluídos, tendo em vistas que os sistemas de informações atualizados e a testagem de amostras da população são importantes para gerar as informações e os indicadores necessários para subsidiar a tomada de decisão e monitorar o impacto das ações de controle, estimar a proporção de protegidos na população, subsidiando para a definição das melhores estratégias em saúde, como para a suspensão ou retomada gradual das atividades.

O plano de contingência orienta capacitar os profissionais de saúde para o enfrentamento da pandemia, destacando: detecção de possíveis casos suspeitos, fluxo de notificação dos casos, coleta e encaminhamento de amostra, atuação diante de casos suspeitos, medidas de biossegurança, sensibilização da etiqueta respiratória, assim como prover e distribuir insumos às equipes da APS, garantir insumos para realização de exames diagnósticos e outros recursos necessários para operacionalização da coleta, acondicionamento e transporte das amostras.

A SESA, enquanto coordenadora da política do SUS no Estado, fomenta a integralidade das ações na prevenção e monitoramento da doença, bem como na assistência à saúde da população. As ações em andamento e as que serão implementadas devem promover a assistência adequada às pessoas, com sensível e oportuna atuação da vigilância em saúde, bem como ações de informação e comunicação.

Desta maneira, estas diretrizes formuladas e integralizadas no plano de contingência têm por objetivo colaborar com os serviços de saúde na mitigação dos processos epidêmicos, comunicação de risco e na redução da morbimortalidade por Covid-19. Contudo, existe a necessidade de mais estudos para evidenciar avanços e revisões nos planos diversos elaborados por secretarias e seus comitês.

Ademais, elaborar, executar e revisar o plano de contingência possibilitou pensar na realidade, que por sua vez embasou as ações prioritárias descri- 
tas, corroborando a garantia dos atributos da APS e consequentemente o seu fortalecimento.

\section{CONSIDERAÇÕES FINAIS}

Vivencia-se a mais importante pandemia da história mundial recente causada por um novo coronavírus (SARS-CoV-2), com significativo impacto na economia, na saúde pública e na saúde mental de toda a sociedade, principalmente dos mais vulneráveis.

Diante desse risco, configurado na doença Covid-19, a elaboração e atualização do plano de contingência foram fundamentais na organização das ações de intervenção na realidade e geração de respostas apropriadas a essa pandemia.

A participação na elaboração do plano de contingência estadual pela equipe da APS garantiu visibilidade nesse enfrentamento, bem como a priorização de ações na estruturação dos pontos norteadores que sustentam a APS.

Desse modo, a APS que se quer construir socialmente é uma estratégia resolutiva, com capacidade de coordenar a Rede de Atenção à Saúde (RAS) e com responsabilidades claras, sanitárias e econômicas da população adstrita.

\section{AGRADECIMENTOS}

Agradecemos à Elaine Drehmer de Almeida Cruz, apoiadora pela OPAS junto à SESA-PR, pelas contribuições ao texto. 


\section{REFERÊNCIAS}

1. World Health Organization (WHO). Coronavirus Disease (COVID-19) Dashboard [Internet]. Geneva: WHO; 2020. [citado em 2020 Jun 23]. Disponível em: https://covid19.who.int/.

2. Sarti TD, Lazarini WS, Fontenelle LF, Almeida APS. Qual o papel da Atenção Primária à Saúde diante da pandemia provocada pela COVID-19? [Internet]. Epidemiol. Serv. Saúde; 2020. [citado em 2020 Jun 16]; 9(2). Disponível em: https://www.scielo.br/ scielo.php?script=sci_arttext\&pid=\$2237-96222020000200903\&lng=pt\&nrm=iso\&tlng=pt.

3. Brasil. Ministério da Saúde (MS). Guia Orientador para o enfrentamento da pandemia Covid-19 na Rede de Atenção à Saúde [Internet]. Brasília: Ministério da Saúde; 2020. [citado em 2020 Jun 16]. Disponível em: http://www.conasems.org.br/ wp- content/uploads/2020/05/Instrumento-Orientador-Conass-Conasems-VERS\%C3\%830-FINAL-3.pdf.

4. Tasca R, Massuda A, Carvalho WM, Buchweitz C, Harzheim E. Recomendações para o fortalecimento da atenção primária à saúde no Brasil [Internet]. Rev Panam Salud Publica; 2020. [citado em 2020 Jun 15]; 44(4). Disponível em: https://iris.paho. org/bitstream/handle/10665.2/51793/v43e1842019.pdf?sequence=1\&isAllowed=y.

5. Starfield B, Shi L, Macinko J. Contribution of primary care to health systems and health. Milbank Q. [Internet]. 2005. [citado em 2020 Jun 15]; 83(3). Disponível em: https://doi.org/10.1111/j.1468-0009.2005.00409.x.

6. Brasil. Ministério da Saúde (MS). Protocolo de manejo clínico do coronavírus (Covid-19) na Atenção Primária à Saúde [Internet]. Brasília: Ministério da Saúde; 2020. [citado em 2020 Jun 15]. Disponível em: https://portaldeboaspraticas.iff. fiocruz.br/wp-content/uploads/2020/05/20200504_ProtocoloManejo_ver09.pdf.

7. Secretaria de Estado da Saúde do Paraná (BR). Plano de resposta a emergências em saúde pública do estado do Paraná. [Internet]. Curitiba: Secretaria de Estado da Saúde do Paraná; 2020. [citado em 2020 Jun 23]. Disponível em: http://www. saude.pr.gov.br/sites/default/arquivos_restritos/files/documento/2020-04/planoderespostaespfinal310718.pdf.

8. Secretaria de Estado da Saúde do Paraná (PR). Resolução SESA Nº126/2020. Ativa o Comitê de Operações de Emergência em Saúde Pública (COE-COVID-19). [Internet]. Curitiba: Secretaria de Estado da Saúde do Paraná; 2020. [citado em 2020 Jun 23]. Disponível em: http://www.saude.pr.gov.br/sites/default/arquivos_restritos/files/documento/2020-04/126_20.pdf.

9. Secretaria de Estado da Saúde do Paraná (PR). Plano de Contingência do Paraná COVID-19 [Internet]. Curitiba: Secretaria de Estado da Saúde do Paraná; 2020. [citado em 2020 Jun 23]. Disponível em: https://portalarquivos.saude.gov.br/images/ pdf/2020/fevereiro/13/PLANO-DE-CONTINGENCIA-novo-coronavirus-PARAN---EM-REVIS--O.pdf.

10. Secretaria de Estado da Saúde do Paraná (PR). Resolução SESA n 317/2020. Aplicação da composição e atribuições do Centro de Operações de Emergência em Saúde Pública (COE-COVID-19) [Internet]. Curitiba: Secretaria de Estado da Saúde do Paraná; 2020. [citado em 2020 Jun 23]. Disponível em: http://www.saude.pr.gov.br/sites/default/arquivos_restritos/files/ documento/2020-04/317_20.pdf.

11. Secretaria de Estado da Saúde do Paraná (PR). Plano de Contingência do Paraná COVID-19 [Internet]. Curitiba: Secretaria de Estado da Saúde do Paraná; 2020. [citado em 2020 Jun 23]. Disponível em: http://www.saude.pr.gov.br/sites/default/ arquivos_restritos/files/documento/2020-06/plano_de_contingencia_do_parana_covid_19_nivel_3_atualizado.pdf.

\section{RECEBIDO: $11 / 08 / 2020$ \\ ACEITO: 07/10/2020}

